\title{
Numerical computation for solving fuzzy differential equations
}

\author{
Fuziyah Ishak, Najihah Chaini \\ Faculty of Computer and Mathematical Sciences, Universiti Teknologi MARA, 40450 Shah Alam, Selangor, Malaysia
}

\begin{tabular}{l} 
Article Info \\
\hline Article history: \\
Received Jan 13, 2019 \\
Revised Mar 17, 2019 \\
Accepted Apr 11, 2019 \\
\hline
\end{tabular}

\section{Keywords:}

Euler method

Extended Trapezoidal method

Fuzzy differential equations

Numerical solution

Trapezoidal method

\begin{abstract}
Fuzzy differential equations (FDEs) play important roles in modeling dynamic systems in science, economics and engineering. The modeling roles are important because most problems in nature are indistinct and uncertain. Numerical methods are needed to solve FDEs since it is difficult to obtain exact solutions. Many approaches have been studied and explored by previous researchers to solve FDEs numerically. Most FDEs are solved by adapting numerical solutions of ordinary differential equations. In this study, we propose the extended Trapezoidal method to solve first order initial value problems of FDEs. The computed results are compared to that of Euler and Trapezoidal methods in terms of errors in order to test the accuracy and validity of the proposed method. The results shown that the extended Trapezoidal method is more accurate in terms of absolute error. Since the extended Trapezoidal method has shown to be an efficient method to solve FDEs, this brings an idea for future researchers to explore and improve the existing numerical methods for solving more general FDEs.
\end{abstract}

Copyright $\odot 2019$ Institute of Advanced Engineering and Science. All rights reserved.

\section{Corresponding Author:}

Fuziyah Ishak, Faculty of Computer and Mathematical Sciences, Universiti Teknologi MARA, 40450 Shah Alam, Selangor, Malaysia. Email: fuziyah@fskm.uitm.edu.my

\section{INTRODUCTION}

The concept of a crisp set is fundamental in mathematics. According to [1], a crisp set is a set that consists of elements with either full or no membership in the set. The principle notion in set theory can be defined as element assigned with value of either 1 or 0 to represent whether it belongs or does not belong to the set respectively. For example, the questions, 'is the woman tall?', and 'is the man handsome?' in crisp set can be answered with yes (1) or no (0). Lotfi Zadeh in [2], a mathematician by profession, first introduced the idea of fuzzy mathematical framework in 1965 which defined it as a class of objects that involves partial set membership. It is called as fuzzy theory, which is a reasonable tool for modeling imprecision and ambiguity in mathematical models. The theory appears in many disciplines including medicine, science and engineering. It is also very useful in decision making process that involves human perception. According to [2], a fuzzy set is a set that allows the membership function to take any value in the interval of Uncertainty level of a fuzzy set can be measured by using the level of fuzziness. The measure of fuzziness is represented by the function such that where is the set gathering all subsets of the universal set and is the real number domain.

The study of fuzzy differential equations (FDEs) is considered as a new branch of fuzzy mathematics. Modeling with FDEs is more relevant and appropriate in representing uncertain systems. The researches in FDEs have been rapidly growing over the last few decades. Chang and Zadeh in [3] first introduced the concept of fuzzy derivative. It has been used as conditions to solve fuzzy problems. Meanwhile, [4] introduced concepts that are related to differential equations. Based on the extension principle of Zadeh, the researchers defined the concepts of fuzzy derivative where the differentiation of 
ordinary functions at a fuzzy point and fuzzy-valued functions at a non-fuzzy point. This approach satisfies the generalized Lipschitz condition in which the fuzzy initial-value problems (IVPs) have specific solution.

Finding solutions for FDEs is very fundamental. Hukuhara differentiability is the most popular approach in solving fuzzy valued functions. Kaleva in [5] has studied further on the concepts of fuzzy-setvalued mappings of a real variable, which the values are normal, convex, upper semi continuous, and support fuzzy sets. Most FDEs can be solved using several approaches, either numerically or analytically. However, for some FDEs, it is difficult to find analytical solutions. Numerical method is an effective way to solve FDEs. According to [6], numerical solution of FDEs is obtained by extending the current classical methods to the fuzzy version of differential equations. Finding actual solutions for fuzzy IVPs can be quite difficult and at times are almost impossible to obtain. In order to overcome these problems, most researchers employ numerical methods for solving the IVPs which can be made as accurately as possible. Conventionally, most of the numerical methods for solving fuzzy IVPS are adapted from that of the numerical methods for solving ODEs. Researchers such as [7-13] have worked on various one-step methods. Meanwhile, authors in [14-17] have worked on family of Adam-Bashforth_Moulton methods.

Since FDEs are applicable in many real life problems, researchers still need to improve and develop numerical methods in order to find better solutions for FDEs. More researchers in [18-25] have also proposed various methods to solve FDEs numerically. This study aims to improve the accuracy of the numerical solution of FDEs. Trapezoidal method has been seen to be able to solve FDEs but current practice has less accuracy with error in approximating the solution for large step-size. We propose extended Trapezoidal method to solve first-order IVPs of linear FDEs numerically. The results are expected to be more accurate as compared to the existing methods.

The organization of the paper is as follows. In Section 2, we discuss some fundamental concepts of fuzzy mathematics and the development of the proposed method for solving FDEs. Selected numerical results are presented and discussed in Section 3. Finally, the conclusions will be presented in Section 4.

\section{RESEARCH METHOD} from [26].

In this section, we introduce some relevant definitions and preliminaries regarding FDEs, as taken

Definition 1. Let $U$ be a nonempty universe and a fuzzy set $A$ in $U$ is a function where $A(x)$ is the degree of membership of $x$ in $A$. The element $x$ will belong more to $A$ if the value of $A(x)$ is closer to value 1 and $x$ will belong less to $A$ if $A(x)$ closer to 0 .

Definition 2. Fuzzy set $A$ in $U$ is presented as a set of ordered pairs of a generic element $x$ and its membership value, $A=\{(x, A(x)) \mid x \in U\}$.

Definition 3. Let $A$ be a fuzzy set in $U$. The support of $A$ is the crisp set of all elements in $U$ with nonzero membership in $A$, i.e.

$$
\operatorname{supp}(A)=\{x \in U \mid A(x)>0\}
$$

Definition 4. Let $A$ be a fuzzy set in $U$. The core of $A$ is the crisp set of all elements in $U$ with membership degree in $A$ equal to 1, i.e.

$$
\operatorname{core}(A)=\{x \in U \mid A(x)=1\} \text {. }
$$

Definition 5. Let $A$ be a fuzzy set defined in $\mathfrak{R}$. $A$ is called a fuzzy interval if

a) $\quad A$ is normal: there exists $x_{0} \in \mathfrak{R}$ such that $A\left(x_{0}\right)=1$;

b) $\quad A$ is convex: for all $x, y \in \mathfrak{R}$ and $0 \leq \lambda \leq 1$, it holds that $A(\lambda x+(1-\lambda) y) \geq \min (A(x), A(y))$;

c) $\quad A$ is upper semi-continuous: for any $x_{0} \in \mathfrak{R}$, it holds that $A\left(x_{0}\right) \geq \lim _{x \rightarrow x_{0}^{ \pm}} A(x)$;

d) $[A]_{0}=\overline{\{x \in \mathfrak{R} \mid A(x) \geq r\}}$ is a compact subset of $\mathfrak{R}$.

Definition 6. The r-level set

$$
[v]_{r}=\{s \mid v(s) \geq r\}, \quad 0<r \leq 1,
$$

is a closed bounded interval, denoted by 


$$
[v]_{r}=\left[v_{1}(r), v_{2}(r)\right] .
$$

Let $I$ be a real interval. A mapping $y: I \rightarrow E$ is called a fuzzy process, and its r-level set is denoted by

$$
[y(t)]_{r}=\left[y_{1}(t ; r), y_{2}(t ; r)\right], \quad t \in I, \quad r \in(0,1] .
$$

Definition 7. Fuzzy number is a function $u: \Re \rightarrow[0,1]$ with the following properties:

a) $\quad u$ is upper semicontinuous on $\Re$,

b) $u(x)=0$ outside of some interval $[c, d]$,

c) there are the real numbers $a$ and $b$ with $c \leq a \leq b \leq d$, such that $u$ is increasing on $[c, a]$, decreasing on $[b, d]$ and $u(x)=1$ for each $x \in[a, b]$,

d) $\quad u$ is fuzzy convex set.

Definition 8. A triangular fuzzy number is denoted by $A$ is defined by $(\alpha, \beta, \gamma)$ where the membership function

$$
\mu_{A}(x)=\left\{\begin{array}{cc}
0, & x<\alpha, \\
\frac{x-\alpha}{\beta-\alpha}, & \alpha \leq x<\beta, \\
\frac{\gamma-x}{\gamma-\beta}, & \beta \leq x \leq \gamma, \\
0, & x>\gamma .
\end{array}\right.
$$

The graphical representation of triangular fuzzy number is given in Figure 1 with $\mu_{A}(\beta)=1$. The r-cut of $A=(\alpha, \beta, \gamma)$ is given by $A_{r}=[\alpha+r(\beta-\alpha), \gamma-r(\gamma-\beta)], \forall r \in[0,1]$.

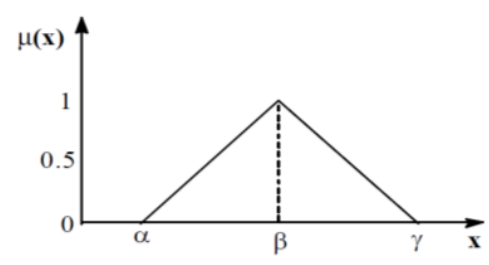

Figure 1. Triangular fuzzy number

Any crisp function can be extended to take on fuzzy set as its argument by applying Zadeh's extension principle, see [2].

In this paper, we consider first-order FDEs $y^{\prime}=f(t, y)$, where $y$ is a fuzzy function of $t, f(t, y)$ is a fuzzy function of crisp variable $t$ and fuzzy variable $y$, and $y^{\prime}$ is Hukuhara or Seikkala fuzzy derivative of $y$. The fuzzy IVPs of first-order is written as follows:

$$
\left\{\begin{array}{l}
y^{\prime}(t)=f(t, y(t)), \\
y(0)=y_{0},
\end{array} \quad t \in[0, T],\right.
$$

where $f: \Re_{+} \times \mathfrak{R} \rightarrow \mathfrak{R}$ is a continuous mapping and $y_{0} \in F(\mathfrak{R})$ with $r$-level sets

$$
\left[y_{0}\right]_{r}=\left[y_{1}(0 ; r), y_{2}(0 ; r)\right], \quad 0<r \leq 1 .
$$

When $y=y(t)$ is a fuzzy number, the extension principle of Zadeh leads to the following supremum definition: 


$$
f(t, y)(s)=\sup \{y(\tau): s=f(t, \tau)\}, \quad s \in \mathfrak{R} .
$$

It follows that

$$
[f(t, y)]_{r}=\left[f_{1}(t, y ; r), f_{2}(t, y ; r)\right], \quad 0<r \leq 1,
$$

Where

$$
\begin{array}{ll}
f_{1}(t, y ; r)=\min \left\{f(t, w): w \in\left[y_{1}(r), y_{2}(r)\right]\right\}, & 0<r \leq 1, \\
f_{2}(t, y ; r)=\max \left\{f(t, w): w \in\left[y_{1}(r), y_{2}(r)\right]\right\}, & 0<r \leq 1 .
\end{array}
$$

In this study, the extended Trapezoidal method is proposed by improving Trapezoidal method and extending one-step further to give more accurate approximate results. The proposed method will be tested on some IVPs to check the accuracy and the validity of the method. Referring to [27], Trapezoidal method for the initial-value problem of ODEs such as

$$
\left\{\begin{array}{l}
y^{\prime}(t)=f(t, y(t)), \\
y(a)=y_{0},
\end{array} \quad a \leq t \leq b,\right.
$$

is given by

$$
y_{n+1}=y_{n}+\frac{h}{2}\left[f_{n}+\hat{f}_{n+1}\right]
$$

where $f_{n}=f\left(t_{n}, y_{n}\right)$ and $\hat{f}_{n+1}=\hat{f}\left(t_{n+1}, \hat{y}_{n+1}\right)$. The notation $y_{n}$ is the approximate to $y\left(t_{n}\right)$, i.e $y_{n} \approx y\left(t_{n}\right)$. Furthermore, the notation $\hat{y}_{n+1}$ refers to the predictor for $y_{n+1}$. The stepsize $h=(b-a) / N$ where $N$ is an integer. The extended Trapezoidal method for the solution (2) is given by

$$
y_{n+1}=y_{n}+h\left[\alpha_{0} f_{n}+\alpha_{1} \hat{f}_{n+1}+\alpha_{2} \hat{f}_{n+2}\right]
$$

Where

$$
\hat{f}_{n+2}=\hat{f}\left(t_{n+2}, \hat{y}_{n+2}\right)
$$

which $\hat{y}_{n+2}$ is a predictor given by

$$
\hat{y}_{n+2}=\beta_{0} y_{n}+\beta_{1} \hat{y}_{n+1}+h\left[\beta_{2} f_{n}+\beta_{3} \hat{f}_{n+1}\right] .
$$

where $\beta_{0}=5, \beta_{1}=-4, \beta_{2}=2, \beta_{3}=4$ and hence

$$
\hat{y}_{n+2}=5 y_{n}-4 \hat{y}_{n+1}+h\left[2 f_{n}+4 \hat{f}_{n+1}\right] \text {. }
$$

Solving (4), we have $\alpha_{0}=\frac{5}{12}, \alpha_{1}=\frac{2}{3}, \alpha_{2}=-\frac{1}{12}$ and hence

$$
y_{n+1}=y_{n}+\frac{h}{12}\left[5 f_{n}+8 \hat{f}_{n+1}-\hat{f}_{n+2}\right]
$$

form the basis of extended Trapezoidal method.

Now, consider the fuzzy initial-value problem (1), 


$$
\left\{\begin{array}{l}
y^{\prime}(t)=f(t, y(t)), \quad t \in[0, T] \\
y(0)=y_{0}
\end{array}\right.
$$

Let the exact solution $\left[Y\left(t_{n+1}\right)\right]_{r}=\left[Y_{1}\left(t_{n+1} ; r\right), Y_{2}\left(t_{n+1} ; r\right)\right]$ is approximated by some

$$
\left[y\left(t_{n+1}\right)\right]_{r}=\left[y_{1}\left(t_{n+1} ; r\right), y_{2}\left(t_{n+1} ; r\right)\right]
$$

Where

$$
y_{1}\left(t_{n+1} ; r\right)=y_{1}\left(t_{n} ; r\right)+\frac{h}{12}\left[5 f\left(t_{n}, y_{1}\left(t_{n} ; r\right)\right)+8 \hat{f}\left(t_{n+1}, \hat{y}_{1}\left(t_{n+1} ; r\right)\right)-\hat{f}\left(t_{n+2}, \hat{y}_{1}\left(t_{n+2} ; r\right)\right)\right]
$$

And

$$
y_{2}\left(t_{n+1} ; r\right)=y_{2}\left(t_{n} ; r\right)+\frac{h}{12}\left[5 f\left(t_{n}, y_{2}\left(t_{n} ; r\right)\right)+8 \hat{f}\left(t_{n+1}, \hat{y}_{2}\left(t_{n+1} ; r\right)\right)-\hat{f}\left(t_{n+2}, \hat{y}_{2}\left(t_{n+2} ; r\right)\right)\right]
$$

as a corrector method. We refer to $\hat{y}_{1}^{(i+1)}\left(t_{n+1} ; r\right)$ as the $i$-th iteration and the predictors are obtained recursively as follows,

$$
\begin{aligned}
& \hat{y}_{1}^{(0)}\left(t_{n+1} ; r\right)=y_{1}\left(t_{n} ; r\right)+h f\left(t_{n}, y_{1}\left(t_{n} ; r\right)\right), \\
& \hat{y}_{1}^{(1)}\left(t_{n+1} ; r\right)=y_{1}\left(t_{n} ; r\right)+\frac{h}{2}\left[f\left(t_{n}, y_{1}\left(t_{n} ; r\right)\right)+f\left(t_{n+1}, \hat{y}_{1}^{(0)}\left(t_{n+1} ; r\right)\right)\right], \\
& \hat{y}_{1}^{(2)}\left(t_{n+1} ; r\right)=y_{1}\left(t_{n} ; r\right)+\frac{h}{2}\left[f\left(t_{n}, y_{1}\left(t_{n} ; r\right)\right)+f\left(t_{n+1}, \hat{y}_{1}^{(1)}\left(t_{n+1} ; r\right)\right)\right], \\
& \hat{y}_{1}^{(3)}\left(t_{n+1} ; r\right)=y_{1}\left(t_{n} ; r\right)+\frac{h}{2}\left[f\left(t_{n}, y_{1}\left(t_{n} ; r\right)\right)+f\left(t_{n+1}, \hat{y}_{1}^{(2)}\left(t_{n+1} ; r\right)\right)\right] .
\end{aligned}
$$

After performing three iterations, and since no further changes occur in the value of $\hat{y}_{1}^{(3)}\left(t_{n+1} ; r\right)$, we take $\hat{y}_{1}^{(3)}\left(t_{n+1} ; r\right)$, to be $\hat{y}_{1}\left(t_{n+1} ; r\right)$. Therefore,

$$
\hat{y}_{1}\left(t_{n+2} ; r\right)=5 y_{1}\left(t_{n} ; r\right)-4 \hat{y}_{1}\left(t_{n+1} ; r\right)+h\left[2 f\left(t_{n}, y_{1}\left(t_{n} ; r\right)\right)+4 \hat{f}\left(t_{n+1}, \hat{y}_{1}\left(t_{n+1} ; r\right)\right)\right] .
$$

Similarly,

$$
\begin{aligned}
& \hat{y}_{2}^{(0)}\left(t_{n+1} ; r\right)=y_{2}\left(t_{n} ; r\right)+h f\left(t_{n}, y_{2}\left(t_{n} ; r\right)\right), \\
& \hat{y}_{2}^{(1)}\left(t_{n+1} ; r\right)=y_{2}\left(t_{n} ; r\right)+\frac{h}{2}\left[f\left(t_{n}, y_{2}\left(t_{n} ; r\right)\right)+f\left(t_{n+1}, \hat{y}_{2}^{(0)}\left(t_{n+1} ; r\right)\right)\right], \\
& \hat{y}_{2}^{(2)}\left(t_{n+1} ; r\right)=y_{2}\left(t_{n} ; r\right)+\frac{h}{2}\left[f\left(t_{n}, y_{2}\left(t_{n} ; r\right)\right)+f\left(t_{n+1}, \hat{y}_{2}^{(1)}\left(t_{n+1} ; r\right)\right)\right], \\
& \hat{y}_{2}^{(3)}\left(t_{n+1} ; r\right)=y_{2}\left(t_{n} ; r\right)+\frac{h}{2}\left[f\left(t_{n}, y_{2}\left(t_{n} ; r\right)\right)+f\left(t_{n+1}, \hat{y}_{2}^{(2)}\left(t_{n+1} ; r\right)\right)\right] .
\end{aligned}
$$

For the same reasons as the above, after three iterations, we let $\hat{y}_{2}\left(t_{n+1} ; r\right)=\hat{y}_{2}^{(3)}\left(t_{n+1} ; r\right)$. Thus, 


$$
\hat{y}_{1}\left(t_{n+2} ; r\right)=5 y_{1}\left(t_{n} ; r\right)-4 \hat{y}_{1}\left(t_{n+1} ; r\right)+h\left[2 f\left(t_{n}, y_{1}\left(t_{n} ; r\right)\right)+4 \hat{f}\left(t_{n+1}, \hat{y}_{1}\left(t_{n+1} ; r\right)\right)\right]
$$

Moreover, the exact and approximate solutions at $t_{n+1}, 0 \leq n \leq N$ are denoted by $\left[Y\left(t_{n+1}\right)\right]_{r}=\left[Y_{1}\left(t_{n+1} ; r\right) ; Y_{2}\left(t_{n+1} ; r\right)\right] \quad$ and $\quad\left[y\left(t_{n+1}\right)\right]_{r}=\left[y_{1}\left(t_{n+1} ; r\right), y_{2}\left(t_{n+1} ; r\right)\right] \quad$ respectively. Figure 2 represents the algorithm for the implementation of extended trapezoidal method for solving fuzzy IVPs.

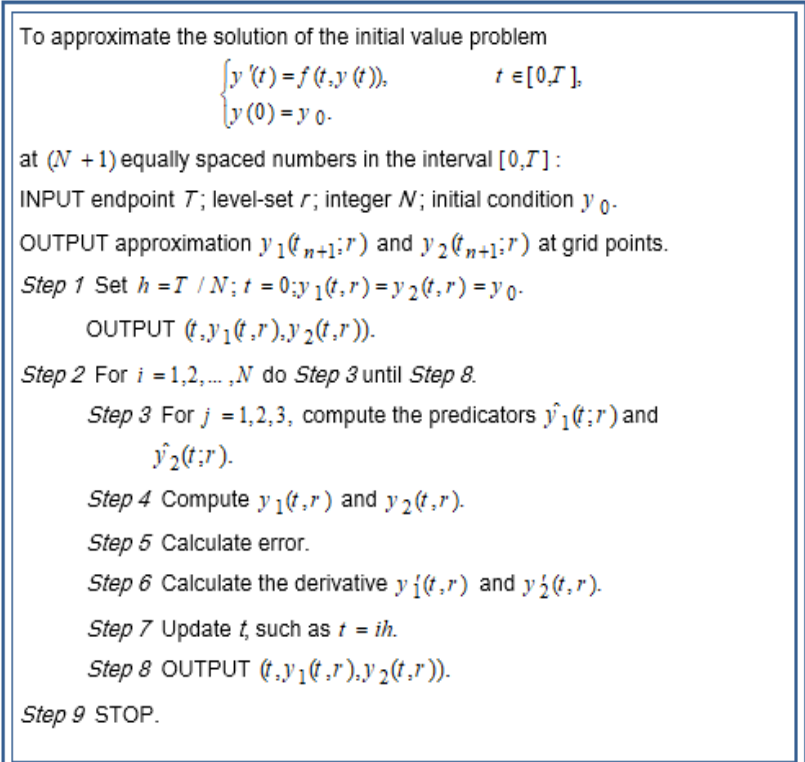

Figure 2. Algorithm for solving fuzzy IVPs

\section{RESULTS AND DISCUSSION}

In testing the validity and accuracy of the implemented method, various first order IVPs are solved using the proposed method with various values of stepsizes. In this section, we illustrate numerical results for one test problem using two different stepsizes. The results of extended Trapezoidal method will be compared to the existing Euler and Trapezoidal methods. For more numerical results on various test problems, kindly refer to [28].

Test Problem 1

Consider the following fuzzy IVPs taken from [11]:

$$
\begin{array}{ll}
y^{\prime}(t)=y(t), & t \in[0,1], \\
y(0)=(0.75+0.25 r, 1.125-0.125 r), & 0<r \leq 1 .
\end{array}
$$

Exact solution at $t=1$ is given by

$$
Y(1 ; r)=[(0.75+0.25 r) e,(1.125-0.125 r) e], \quad 0<r \leq 1 .
$$

The numerical results in terms of absolute errors for test Problem 1 by using Euler, Trapezoidal and extended Trapezoidal method with step-sizes $h=0.1$ and $h=0.0001$ are presented in Table 1 and Table 2 respectively. The exact solutions and approximate solutions are compared and illustrated in Figure 3.

The numerical results in terms of absolute errors for test Problem 1 by using Euler, Trapezoidal and extended Trapezoidal method with step-sizes and are presented in Table 1 and Table 2 respectively. The exact solutions and approximate solutions are compared and illustrated in Figure 3.

From the numerical results, it is clearly seen that the error becomes smaller as the stepsize decreases. For each step-size, the error in extended Trapezoidal method is smaller compared to the errors in Euler method and Trapezoidal method. Thus, it is concluded that the proposed method gives better accuracy when solving Test Problem 1. The exact and approximate solutions for Test Problem 1 at with and compared and 
plotted in Figure 3. In the figure, EM means Euler method, TM is Trapezoidal method, ETM refers to extended Trapezoidal method and Exact means exact solution at From Figure 3, it is clearly seen that the errors for extended Trapezoidal method are very small as compared to the existing method. As stepsize decreases, other tested methods also obtain comparable results in terms of accuracy. Thus, it can be concluded that extended Trapezoidal method is very suitable in solving FDEs numerically.

Table 1. Absolute Error of Test Problem 1 for

\begin{tabular}{|c|c|c|c|c|c|c|}
\hline \multirow[b]{2}{*}{$r$} & \multicolumn{2}{|c|}{ Euler Method } & \multicolumn{2}{|c|}{ Trapezoidal Method } & \multicolumn{2}{|c|}{ Extended Trapezoidal Method } \\
\hline & $y_{1}\left(t_{N} ; r\right)$ & $y_{2}\left(t_{N} ; r\right)$ & $y_{1}\left(t_{N} ; r\right)$ & $y_{2}\left(t_{N} ; r\right)$ & $y_{1}\left(t_{N} ; r\right)$ & $y_{2}\left(t_{N} ; r\right)$ \\
\hline 0.1 & $9.65180 \mathrm{e}-02$ & $1.38550 \mathrm{e}-01$ & $3.25576 \mathrm{e}-03$ & $4.67359 \mathrm{e}-03$ & $8.49591 \mathrm{e}-05$ & $1.21957 \mathrm{e}-04$ \\
\hline 0.2 & $9.96315 \mathrm{e}-02$ & $1.36993 \mathrm{e}-01$ & $3.36079 \mathrm{e}-03$ & $4.62108 \mathrm{e}-03$ & $8.76997 \mathrm{e}-05$ & $1.20587 \mathrm{e}-04$ \\
\hline 0.3 & $1.02745 \mathrm{e}-01$ & $1.35437 \mathrm{e}-01$ & $3.46581 \mathrm{e}-03$ & $4.56857 \mathrm{e}-03$ & $9.04403 \mathrm{e}-05$ & $1.19217 \mathrm{e}-04$ \\
\hline 0.4 & $1.05858 \mathrm{e}-01$ & $1.33880 \mathrm{e}-01$ & $3.57083 \mathrm{e}-03$ & $4.51606 \mathrm{e}-03$ & $9.31809 \mathrm{e}-05$ & $1.17846 \mathrm{e}-04$ \\
\hline 0.5 & $1.08972 \mathrm{e}-01$ & $1.32323 \mathrm{e}-01$ & $3.67586 \mathrm{e}-03$ & $4.46354 \mathrm{e}-03$ & $9.59216 \mathrm{e}-05$ & $1.16476 \mathrm{e}-04$ \\
\hline 0.6 & $1.12085 \mathrm{e}-01$ & $1.30766 \mathrm{e}-01$ & $3.78088 \mathrm{e}-03$ & $4.41103 \mathrm{e}-03$ & $9.86622 \mathrm{e}-05$ & $1.15106 \mathrm{e}-04$ \\
\hline 0.7 & $1.15199 \mathrm{e}-01$ & $1.29210 \mathrm{e}-01$ & $3.88591 \mathrm{e}-03$ & $4.35852 \mathrm{e}-03$ & $1.01403 \mathrm{e}-04$ & $1.13736 \mathrm{e}-04$ \\
\hline 0.8 & $1.18312 \mathrm{e}-01$ & $1.27653 \mathrm{e}-01$ & $3.99093 \mathrm{e}-03$ & $4.30601 \mathrm{e}-03$ & $1.04143 \mathrm{e}-04$ & $1.12365 \mathrm{e}-04$ \\
\hline 0.9 & $1.21426 \mathrm{e}-01$ & $1.26096 \mathrm{e}-01$ & $4.09596 \mathrm{e}-03$ & $4.25349 \mathrm{e}-03$ & $1.06884 \mathrm{e}-04$ & $1.10995 \mathrm{e}-04$ \\
\hline 1.0 & $1.24539 \mathrm{e}-01$ & $1.24539 \mathrm{e}-01$ & $4.20098 \mathrm{e}-03$ & $4.20098 \mathrm{e}-03$ & $1.09625 \mathrm{e}-04$ & $1.09625 \mathrm{e}-04$ \\
\hline
\end{tabular}

Note: $8.49591 \mathrm{e}-05$ means $8.49591 \times 10^{-5}$

Table 2. Absolute Error of Problem 1 for $h=0.0001$

\begin{tabular}{|c|c|c|c|c|c|c|}
\hline \multirow[b]{2}{*}{$r$} & \multicolumn{2}{|c|}{ Euler Method } & \multicolumn{2}{|c|}{ Trapezoidal Method } & \multicolumn{2}{|c|}{ Extended Trapezoidal Method } \\
\hline & $y_{1}\left(t_{N} ; r\right)$ & $y_{2}\left(t_{N} ; r\right)$ & $y_{1}\left(t_{N} ; r\right)$ & $y_{2}\left(t_{N} ; r\right)$ & $y_{1}\left(t_{N} ; r\right)$ & $y_{2}\left(t_{N} ; r\right)$ \\
\hline 0.1 & $1.05324 \mathrm{e}-04$ & $1.51191 \mathrm{e}-04$ & $3.51086 \mathrm{e}-09$ & $5.03980 \mathrm{e}-09$ & $9.81437 \mathrm{e}-14$ & $1.23013 \mathrm{e}-13$ \\
\hline 0.2 & $1.08721 \mathrm{e}-04$ & $1.49492 \mathrm{e}-04$ & $3.62411 \mathrm{e}-09$ & $4.98316 \mathrm{e}-09$ & $8.74856 \mathrm{e}-14$ & $9.54792 \mathrm{e}-14$ \\
\hline 0.3 & $1.12119 \mathrm{e}-04$ & $1.47793 \mathrm{e}-04$ & $3.73736 \mathrm{e}-09$ & $4.92651 \mathrm{e}-09$ & $1.06581 \mathrm{e}-13$ & $1.24345 \mathrm{e}-13$ \\
\hline 0.4 & $1.15516 \mathrm{e}-04$ & $1.46094 \mathrm{e}-04$ & $3.85061 \mathrm{e}-09$ & $4.86987 \mathrm{e}-09$ & $9.76996 \mathrm{e}-14$ & $1.27454 \mathrm{e}-13$ \\
\hline 0.5 & $1.18914 \mathrm{e}-04$ & $1.44395 \mathrm{e}-04$ & $3.96385 \mathrm{e}-09$ & $4.81324 \mathrm{e}-09$ & $7.77156 \mathrm{e}-14$ & $1.27010 \mathrm{e}-13$ \\
\hline 0.6 & $1.22311 \mathrm{e}-04$ & $1.42697 \mathrm{e}-04$ & $4.07713 \mathrm{e}-09$ & $4.75664 \mathrm{e}-09$ & $1.13687 \mathrm{e}-13$ & $1.07470 \mathrm{e}-13$ \\
\hline 0.7 & $1.25709 \mathrm{e}-04$ & $1.40998 \mathrm{e}-04$ & $4.19038 \mathrm{e}-09$ & $4.69998 \mathrm{e}-09$ & $1.00364 \mathrm{e}-13$ & $1.14131 \mathrm{e}-13$ \\
\hline 0.8 & $1.29107 \mathrm{e}-04$ & $1.39299 \mathrm{e}-04$ & $4.30362 \mathrm{e}-09$ & $4.64340 \mathrm{e}-09$ & $1.33671 \mathrm{e}-13$ & $1.23457 \mathrm{e}-13$ \\
\hline 0.9 & $1.32504 \mathrm{e}-04$ & $1.37600 \mathrm{e}-04$ & $4.41689 \mathrm{e}-09$ & $4.58676 \mathrm{e}-09$ & $8.03801 \mathrm{e}-14$ & $1.31894 \mathrm{e}-13$ \\
\hline 1.0 & $1.35902 \mathrm{e}-04$ & $1.35902 \mathrm{e}-04$ & $4.53016 \mathrm{e}-09$ & $4.53016 \mathrm{e}-09$ & $1.35447 \mathrm{e}-13$ & $1.35447 \mathrm{e}-13$ \\
\hline
\end{tabular}

Note: $8.49591 \mathrm{e}-05$ means $8.49591 \times 10^{-5}$

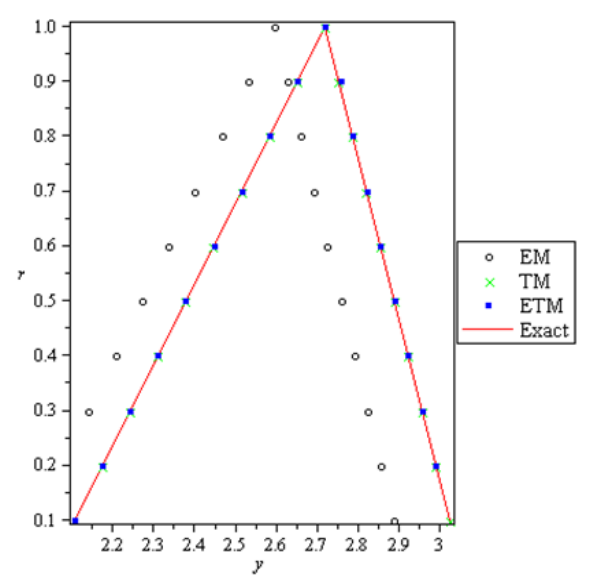

(a) Approximate and Exact Solutions of Test Problem 1 with $h=0.1$

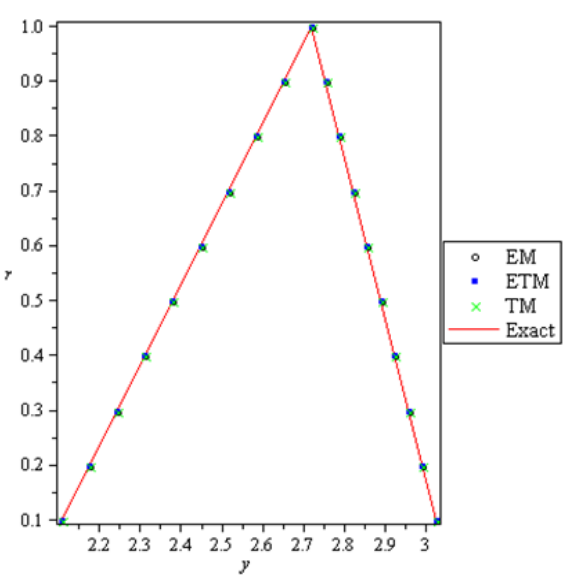

(b) Approximate and Exact Solutions of Test Problem 1 with $h=0.0001$

Figure 3. Approximate and Exact Solutions of Test Problem 1 with $h=0.1$ and $h=0.0001$

\section{CONCLUSION}

We have presented numerical computation for the solutions of fuzzy IVPs. The numerical computation focuses on the implementation of extended Trapezoidal method for solving FDEs. Numerical 
results for extended Trapezoidal methods are compared with the existing Euler and Trapezoidal methods. The proposed extended trapezoidal method performs very well when compared with the existing method of the same order. The errors, computed when different stepsizes being employed, indicate that the numerical solutions approach exact solutions, as the stepsizes are getting smaller. We conclude that extended Trapezoidal method achieves the desired accuracy and therefore, the method is suitable for solving FDEs.

\section{REFERENCES}

[1] H. J. Zimmermann, "Fuzzy Set Theory," Wiley Interdisciplinary Reviews: Computational Statistics, vol. 2(3), pp. 317-332, 2010.

[2] L. A. Zadeh, "Fuzzy Sets," Information and Control, vol. 8, pp. 338-353, 1965.

[3] S. L. Chang and L. A. Zadeh, "On Fuzzy Mapping and Control," IEEE Trans. System Men Cybernet, vol. 2, pp. 2034, 1972

[4] D. Dubois and H. Prade, "Towards Fuzzy Differential Calculus," Fuzzy Sets and System, vol. 8, pp. 225-233, 1982.

[5] O. Kaleva, "Fuzzy Differential Equations," Fuzzy Sets and System, vol. 24(3), pp. 301-317, 1987.

[6] E. Hullermeier, "Numerical Methods for Fuzzy Initial Value Problems," Int, Jour. of Uncertainty Fuzziness Knowledge-based Systems, vol. 7, pp. 439-461, 1999.

[7] M. Ma, et al, "Numerical Solutions of Fuzzy Differential Equations," Fuzzy Sets and Systems, vol. 106, pp. 133138, 1999.

[8] S. C. Palligkinis et al, "Runge Kutta Methods for Fuzzy Differential Equations," Applied Mathematics and Computation, vol. 2019(1), pp. 97-105, 2009.

[9] S. Abbasbandy and T. Allahviranloo, "Numerical Solution of Fuzzy Differential Equation by Third Order Runge Kutta Method," Nonlinear Studies, vol. 1191), pp. 117-129, 2004.

[10] K. Kanagarajan and M. Sambath, "Numerical Solution of Fuzzy Differential Equation by Runge Kutta Method," Int. Jour. of Applied Mathematics and Computation, vol. 2(4), pp. 1-8, 2010.

[11] T. Jayakumar, et al, "Numerical Solution of Fuzzy Differential Equation by Runge Kutta Method of Order Five," Int. Jour. of Applied Mathematical Science, vol. 6, pp. 2986-3002, 2012.

[12] K. Ivaz, et al, "A Numerical Method for Fuzzy Differential Equations and Hybrid Fuzzy Differential Equations," Abstract and Applied Analysis, pp. 1 - 10, 2013.

[13] S. Narayanamoorthy and T. L. Yookesh, "Third Order Runge Kutta Method for Solving Differential Equation in Fuzzy Environment," Int. Jour. of Pure and Applied Mathematics, vol. 101(5), pp. 795-802, 2015.

[14] T. Allahviranloo, "Numerical Solution of Fuzzy Differential Equation by Adams Bashforth Two-Step Method," Journal of Applied Mathematics, pp. 36-47, 2004.

[15] T. Allahviranloo, et al, "Two-Step Method for Fuzzy Differential Equations," International Mathematical Forum, vol 1(17), pp. 823-832, 2006.

[16] T. Allahviranloo, et al, "Numerical Solution of Fuzzy Differential Equations by Predictor Corrector Method," Information Science, vol 177(7), pp. 1633-1647, 2007.

[17] T. Jayakumar, et al, "Numerical Solution of Fuzzy Differential Equations by Adams Fifth Order Predictor Corrector Method," International Jour. of Mathematics Trends and Technology, vol 8, pp. 33-50, 2014.

[18] J. G. Oghonyon, et al, "K-step Block Predictor-Corrector Method for Solving First Order Ordinary Differential Equations," Research Jour. of Applied Sciences, vol 10, no. 11, pp. 779-785, 2015.

[19] Y. Seif and A. Khastan, "A Numerical Method for Solving Fuzzy Differential Equations," International Jour. of Mathematics and Computation, vol 4, no. 4, pp. 81-91, 2011.

[20] R. G. Sharmila and E. C. Amirtharaj, "Numerical Solutions of First Order Fuzzy Initial Value Problems by Nonlinear Trapezoidal Formulae based on Variety of Means," Indian Journal of Research, vol 3, no. 5, 2014.

[21] S. P. Mondal, et al, "System of Differential Equations with Initial Value as Triangular Intuitiosnistic Fuzzy Number and its Application," International Jour. of Applied and Computational Mathematics, vol 1, no 3, pp. 449-474, 2015.

[22] S. P. Mondal, et al, "Numerical Solution of First Order Linear Differential Equations in Fuzzy Environment by Runge-Kutta-Fehlberg Method and its Application," International Jour. of Differential Equations, pp. 1-14, 2016.

[23] B. Ghazanfari and A. Shakerami, "Numerical Solutions of Fuzzy Differential Equations by Extended Runge-Kutta like Formulae of Order Four," Fuzzy Sets and Systems, vol 1889, no. 1, pp. 74-91, 2012.

[24] M. Chehlabi and T. Allahviranloo, "Positive or Negative Solutions to First-Order Fully Linear Differential Equations under Generalized Differentiability," Applied Soft Computing, vol 70, pp. 359-370, 2018.

[25] A. Ahmadian, et al, "Numerical Solutions of Fuzzy Differential Equations by an Efficient Runge-Kutta Method with Generalized Differentiability," Fuzzy Set and Systems, vol 331, pp. 47-67, 2018.

[26] M. Z. Ahmad, et al, "Analytical and Numerical Solutions of Fuzzy Differential Equations," Information Science, vol. 236, pp. 156-167, 2013.

[27] R. A. Usmani and R. P. Agarwal, "An A-Stable Extended Trapezoidal Rule for the Integration of Ordinary Differential Equations," Computers and Mathematics with Applications, vol 11(12), pp. 1183-1191, 1985.

[28] N. Chaini, "Numerical Solution of Fuzzy Differential Equations by Adams Fifth Order Predictor Corrector Method," Extended Trapezoidal Method for Numerical Solution of Fuzzy Differential Equations, Master Dissertation, Universiti Teknologi MARA, 2016. 